\title{
Mercuric Triflate Catalyzed Hydroxylative Carbocyclization of 1,6-Enynes
}

\author{
Mugio Nishizawa, ${ }^{\star}$ Veejendra K. Yadav, ${ }^{\dagger}$ Mariusz Skwarczynski, Hiroko Takao, Hiroshi Imagawa, and Takumichi
} Sugihara

Faculty of Pharmaceutical Sciences, Tokushima Bunri University Yamashiro-cho, Tokushima 770-8514, Japan

General procedure: Mercuric triflate was prepared by adding triflic anhydride to a stirred suspension of yellow mercuric oxide in $\mathrm{CH}_{3} \mathrm{CN}$. To a solution of an enyne $(1 \mathrm{mmol})$ and $\mathrm{H}_{2} \mathrm{O}(5 \mathrm{mmol})$ in $\mathrm{CH}_{3} \mathrm{NO}_{2}(9 \mathrm{~mL})$ was added a $0.1 \mathrm{M}$ solution of $\mathrm{Hg}(\mathrm{OTf})_{2}$ in $\mathrm{CH}_{3} \mathrm{CN}(0.01 \mathrm{mmol})$, and the resulting mixture was stirred for $20 \mathrm{~h}$ at room temperature. The reaction was quenched with saturated aqueous $\mathrm{NaCl}$ and $\mathrm{NaHCO}_{3}(5 \mathrm{~mL}$ each) and the product was extracted into EtOAc that was dried and concentrated. Column chromatography of the crude material with mixtures of hexane and EtOAc afforded the product.

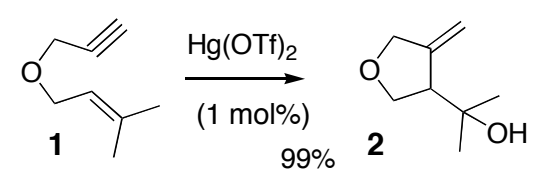

1. ${ }^{1} \mathrm{H}$ NMR $\left(300 \mathrm{MHz}, \mathrm{CDCl}_{3}\right): \delta \quad 5.38-5.31(1 \mathrm{H}, \mathrm{m}), 4.14-4.13(2 \mathrm{H}, \mathrm{dd}, \mathrm{J}=2.4,0.9 \mathrm{~Hz}), 4.08-4.05(2 \mathrm{H}, \mathrm{dd}, \mathrm{J}=7.2,0.3 \mathrm{~Hz}), 2.44-$ $2.42(1 \mathrm{H}, \mathrm{dt}, \mathrm{J}=2.4,0.9 \mathrm{~Hz}), 1.77(3 \mathrm{H}, \mathrm{s}), 1.71(3 \mathrm{H}, \mathrm{s}) .{ }^{13} \mathrm{C} \mathrm{NMR}\left(50 \mathrm{MHz}, \mathrm{CDCl}_{3}\right): \delta$ 138.4, 120.1, 80.0, 74.1, 65.9, 56.7, 25.8, 18.0. Miura, K.; Saito, H.; Fujisawa, N.; Hosomi, A. J. Org. Chem. 2000, 65, 8119-8122.

2. ${ }^{1} \mathrm{H}$ NMR (300 MHz, $\left.\mathrm{CDCl}_{3}\right): \delta$ 5.19-5.16 (1H, q, J = 2,4 Hz), 5.10-5.09 (1H, q, J = 2.1 Hz), 4.37-4.22 (2H, m), 4.03-3.98 (1H, dd, $\mathrm{J}=9.6,3.3 \mathrm{~Hz}), 3.94-3.88(1 \mathrm{H}, \mathrm{dd}, \mathrm{J}=9.6,6.6 \mathrm{~Hz}), 2.71-2.67(1 \mathrm{H}, \mathrm{m}), 1.87(1 \mathrm{H}, \mathrm{bs}), 1.25(3 \mathrm{H}, \mathrm{s}), 1.23(3 \mathrm{H}, \mathrm{s}) .{ }^{13} \mathrm{C} \mathrm{NMR}(50 \mathrm{MHz}$, $\left.\mathrm{CDCl}_{3}\right): \delta 148.8,107.5,71.91,71.88,71.0,54.8,27.3,26.9$. Calcd $\mathrm{m} / \mathrm{z}$ for $\mathrm{C}_{8} \mathrm{H}_{13} \mathrm{O}_{2}[\mathrm{M}-\mathrm{H}]^{+}=141.0916$; observed $\mathrm{m} / \mathrm{z}=141.0899$. Mendez, M.; Munoz, M. P.; Nevado, C.; Cardenas, D. J.; Echavarren, A. M. J. Am. Chem. Soc. 2001, 123, 10511-10520; Mendez, M.; Muniz, M. P.; Echavarren, A. M. J. Am. Chem. Soc. 2000, 122, 11549-11550.

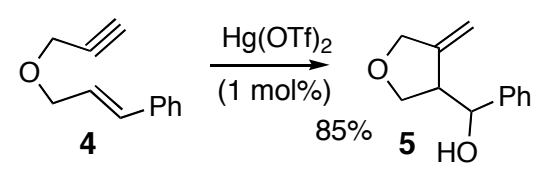

4. ${ }^{1} \mathrm{H}$ NMR (300 MHz, $\left.\mathrm{CDCl}_{3}\right): \delta$ 7.41-7.22 (5H, m), 6.68-6.62 (1H, d, J = 15.9 Hz), 6.33-6.23 (1H, dt, J = 15.9, $\left.6 \mathrm{~Hz}\right), 4.26-4.23$ $(2 \mathrm{H}, \mathrm{dd}, \mathrm{J}=6.3,1.5 \mathrm{~Hz}), 4.21-4.20(2 \mathrm{H}, \mathrm{dd}, \mathrm{J}=2.4,0.6 \mathrm{~Hz}), 2.47-2.45(1 \mathrm{H}, \mathrm{dt}, \mathrm{J}=2.4,0.3 \mathrm{~Hz}) .{ }^{13} \mathrm{C} \mathrm{NMR}\left(75 \mathrm{MHz}, \mathrm{CDCl}_{3}\right): \delta 136.4$, 133.4, 128.5, 127.8, 126.5, 124.9, 79.6, 74.5, 70.2, 57.0. Miura, K.; Saito, H.; Fujisawa, N.; Hosomi, A. J. Org. Chem. 2000, 65, 8119-8122; Trost, B. M.; Edstrom, E. D.; C.-Petillo, M. B. J. Org. Chem. 1989, 54, 4489-4490; Barluenga, J.; Aznar, F.; Liz, R.; Bayod, M. J. Org. Chem. 1987, 52, 5190-5194. 
5. ${ }^{1} \mathrm{H}$ NMR (300 MHz, $\left.\mathrm{CDCl}_{3}\right): \delta$ 7.32-7.22 (5H, m), $4.87(1 \mathrm{H}, \mathrm{d}, \mathrm{J}=2.1 \mathrm{~Hz}), 4.66-4.63(1 \mathrm{H}, \mathrm{d}, \mathrm{J}=6.9 \mathrm{~Hz}), 4.46(1 \mathrm{H}, \mathrm{d}, \mathrm{J}=2.1 \mathrm{~Hz})$, 4.28-4.15 (2H, m), 4.10-4.05 (1H, dd, J = 9, 4.8 Hz), 3.82-3.77 (1H, dd, J = 9, 6.6 Hz), $3.02(1 \mathrm{H}, \mathrm{bs}), 2.98-2.92(1 \mathrm{H}, \mathrm{m}) .{ }^{13} \mathrm{C} \mathrm{NMR}$ $\left(75 \mathrm{MHz}, \mathrm{CDCl}_{3}\right): \delta 147.6,142.4,128.1,127.5,126,4,106.3,74.4,71.6,70.4,50.9$. Calcd m/z for $\mathrm{C}_{12} \mathrm{H}_{13} \mathrm{O}\left[\mathrm{M}-\mathrm{H}_{2} \mathrm{O}+\mathrm{H}^{+}\right]=$ 173.0966; observed m/z = 173.0947. Galland, J.-C.; Dias, S.; Savignac, M.; Genet, J.-P. Tetrahedron 2001, 57, 5137-5148. Mendez, M.; Munoz, M. P.; Nevado, C.; Cardenas, D. J.; Echavarren, A. M. J. Am. Chem. Soc. 2001, 123, 10511-10520; Mendez, M.; Munoz, M. P.; Echavarren, A. M. J. Am. Chem. Soc. 2000, 122, 11549-11550; Galland, J.-C.; Savignac, M.; Genet, J.-P. Tetrahedron Lett. 1997, 38, 8695-8698.

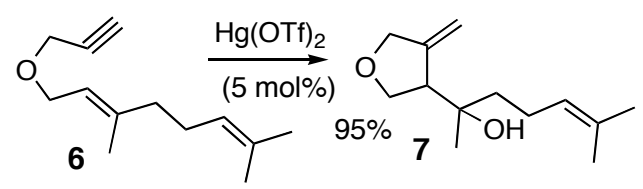

6. ${ }^{1} \mathrm{H}$ NMR (200 MHz, $\left.\mathrm{CDCl}_{3}\right): \delta$ 5.37-5.28 (1H, m), 5.12-5.04 (1H, m), $4.12(2 \mathrm{H}, \mathrm{d}, \mathrm{J}=1.8 \mathrm{~Hz}), 4.08(2 \mathrm{H}, \mathrm{d}, \mathrm{J}=7 \mathrm{~Hz}), 2.41(1 \mathrm{H}, \mathrm{t}, \mathrm{J}$ $=1.8 \mathrm{~Hz}), 2.18-1.98(4 \mathrm{H}, \mathrm{m}), 1.69(3 \mathrm{H}, \mathrm{s}), 1.67(3 \mathrm{H}, \mathrm{s}), 1.59(3 \mathrm{H}, \mathrm{s}) .{ }^{13} \mathrm{C}$ NMR $\left(50 \mathrm{MHz}, \mathrm{CDCl}_{3}\right): \delta 141.6,131.6,123.9,119.8,80.0$, 74.0, 65.8, 56.5, 39.5, 26.2, 25.6, 17.5, 16.3. Chodnekar, M. S.; Pfiffner, A.; Rigassi, N.; Schwieter, U.; Suchy, M. Ger. Offen. 1971, GWXXBX DE 2115673 19711021 (CAN 76:55261, AN 1972:55261); Ger. Offen. 1971, GWXXBX DE 203895819710218 (CAN 75:64037, AN 1971:464037).

7. ${ }^{1} \mathrm{H}$ NMR (300 MHz, $\left.\mathrm{CDCl}_{3}\right): \delta$ 5.13-5.05 (3H, m), 4.33-4.18 (2H, m), 4.11-4.07 (1H, dd, J = 9.3, 3.3 Hz), 3.90-3.85 $(1 \mathrm{H}, \mathrm{dd}, \mathrm{J}=$ 9.3, $6.9 \mathrm{~Hz}), 2.74-2.71(1 \mathrm{H}, \mathrm{m}), 2.17-2.00(2 \mathrm{H}, \mathrm{m}), 1.98(1 \mathrm{H}, \mathrm{bs}), 1.66(3 \mathrm{H}, \mathrm{d}, \mathrm{J}=0.9 \mathrm{~Hz}), 1.61$ (3H, bs), $1.61-1.46(2 \mathrm{H}, \mathrm{m}), 1.17$ (3H, s). ${ }^{13} \mathrm{C} \mathrm{NMR}\left(75 \mathrm{MHz}, \mathrm{CDCl}_{3}\right): \delta 148.6,131.8,124.2,107.3,73.8,72.0,70.6,53.6,39.1,25.6,23.9,22.1$, 17.6. Calcd m/z for $\mathrm{C}_{13} \mathrm{H}_{23} \mathrm{O}_{2}\left[\mathrm{M}+\mathrm{H}^{+}\right]=211.1698 ;$ observed $\mathrm{m} / \mathrm{z}=211.1671$.

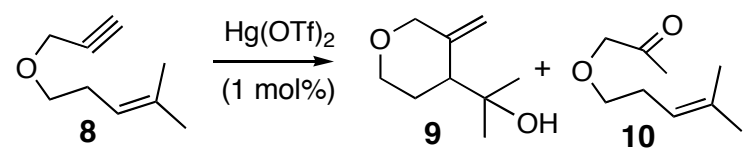

8. ${ }^{1} \mathrm{H}$ NMR $\left(300 \mathrm{MHz}, \mathrm{CDCl}_{3}\right): \quad \delta \quad 5.17-5.10(1 \mathrm{H}, \mathrm{mt}, \mathrm{J}=7.2,1.5 \mathrm{~Hz}), 4.16(2 \mathrm{H}, \mathrm{d}, \mathrm{J}=2.4 \mathrm{~Hz}), 3.51(2 \mathrm{H}, \mathrm{t}, \mathrm{J}=7.2 \mathrm{~Hz}), 2.42(1 \mathrm{H}, \mathrm{t}$, $\mathrm{J}=2.4 \mathrm{~Hz}), 2.35-2.27(2 \mathrm{H}, \mathrm{tq}, \mathrm{J}=7.2,0.9 \mathrm{~Hz}), 1.71(3 \mathrm{H}, \mathrm{d}, \mathrm{J}=0.9 \mathrm{~Hz}), 1.64(3 \mathrm{H}, \mathrm{d}, \mathrm{J}=0.3 \mathrm{~Hz}) .{ }^{13} \mathrm{C} \mathrm{NMR}\left(75 \mathrm{MHz} \mathrm{CDCl}_{3}\right): \delta$ $133.8,120.0,79.9,74.1,69.9,58.0,28.5,25.7,17.8$.

9. ${ }^{1} \mathrm{H}$ NMR (300 MHz, $\left.\mathrm{CDCl}_{3}\right): \delta 5.09(1 \mathrm{H}, \mathrm{bs}), 5.01(1 \mathrm{H}, \mathrm{bs}), 4.24-4.20(1 \mathrm{H}, \mathrm{d}, \mathrm{J}=12 \mathrm{~Hz}), 4.02-3.98(1 \mathrm{H}, \mathrm{d}, \mathrm{J}=12 \mathrm{~Hz}), 3.99-3.94$ $(1 \mathrm{H}, \mathrm{m}), 3.69-3.62(1 \mathrm{H}, \mathrm{m}), 2.35(1 \mathrm{H}, \mathrm{t}, \mathrm{J}=6.3 \mathrm{~Hz}), 1.93-1.84(2 \mathrm{H}, \mathrm{m}), 1.33(3 \mathrm{H}, \mathrm{s}), 1.30(3 \mathrm{H}, \mathrm{s}) .{ }^{13} \mathrm{C} \mathrm{NMR}\left(75 \mathrm{MHz}, \mathrm{CDCl}_{3}\right): \delta$ 143.8, 113.5, 72.9, 72.6, 66.0, 49.9, 29.1, 28.5. Calcd m/z for $\mathrm{C}_{9} \mathrm{H}_{17} \mathrm{O}_{2}[\mathrm{M}+\mathrm{H}]{ }^{+}=157.1229$; observed $\mathrm{m} / \mathrm{z}=157.1205$.

10. ${ }^{1} \mathrm{H}$ NMR (300 MHz, $\left.\mathrm{CDCl}_{3}\right): \delta 5.14-5.07(1 \mathrm{H}, \mathrm{mt}, \mathrm{J}=7.2,1.2 \mathrm{~Hz}), 4.00(2 \mathrm{H}, \mathrm{s}), 3.44(2 \mathrm{H}, \mathrm{t}, \mathrm{J}=7.2 \mathrm{~Hz}), 2.33-2.26(2 \mathrm{H}, \mathrm{q}, \mathrm{J}=6.9$ $\mathrm{Hz}), 2.13(3 \mathrm{H}, \mathrm{s}), 1.67(3 \mathrm{H}, \mathrm{d}, \mathrm{J}=1.2 \mathrm{~Hz}), 1.60(3 \mathrm{H}, \mathrm{bs}) .{ }^{13} \mathrm{C} \mathrm{NMR}\left(75 \mathrm{MHz}, \mathrm{CDCl}_{3}\right): \delta 207.2,133.9,119.8,76.3,71.4,28.5,26.2$, 25.6, 17.7. Calcd $\mathrm{m} / \mathrm{z}$ for $\mathrm{C}_{9} \mathrm{H}_{15} \mathrm{O}_{2}[\mathrm{M}-\mathrm{H}]^{+}=155.1072$; observed $\mathrm{m} / \mathrm{z}=155.1048$. 


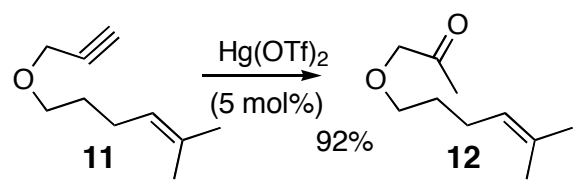

11. ${ }^{1} \mathrm{H}$ NMR (300 MHz, $\left.\mathrm{CDCl}_{3}\right): \delta 5.14-5.07(1 \mathrm{H}, \mathrm{m}), 4.13(2 \mathrm{H}, \mathrm{d}, \mathrm{J}=1.8 \mathrm{~Hz}), 3.50(2 \mathrm{H}, \mathrm{t}, \mathrm{J}=6.5 \mathrm{~Hz}), 2.41(1 \mathrm{H}, \mathrm{t}, \mathrm{J}=1.8 \mathrm{~Hz})$, 2.09-2.01 (2H, q, J = 6.9 Hz), $1.69(3 \mathrm{H}, \mathrm{s}), 1.60(3 \mathrm{H}, \mathrm{s}), 1.68-1.58(2 \mathrm{H}, \mathrm{m}) .{ }^{13} \mathrm{C}$ NMR $\left(75 \mathrm{MHz}, \mathrm{CDCl}_{3}\right): \delta$ 132.0, 123.7, 80.0, 74.0, $69.7,58.0,29.6,25.7,24.4,17.6$.

12. ${ }^{1} \mathrm{H}$ NMR (300 MHz, $\left.\mathrm{CDCl}_{3}\right): \delta$ 5.08-5.03 $(1 \mathrm{H}, \mathrm{m}), 3.96(2 \mathrm{H}, \mathrm{s}), 3.42(2 \mathrm{H}, \mathrm{t}, \mathrm{J}=6.6 \mathrm{~Hz}), 2.11(3 \mathrm{H}, \mathrm{s}), 2.06-1.98(2 \mathrm{H}, \mathrm{q}, \mathrm{J}=7.5$ $\mathrm{Hz}), 1.66-1.58(2 \mathrm{H}, \mathrm{m}), 1.64(3 \mathrm{H}, \mathrm{s}), 1.55(3 \mathrm{H}, \mathrm{s}) .{ }^{13} \mathrm{C} \mathrm{NMR}\left(75 \mathrm{MHz}, \mathrm{CDCl}_{3}\right): \delta 207.2,131.9,123.5,76.3,71.1,29.5,26.2,25.5$, 24.2, 17.5. Calcd $\mathrm{m} / \mathrm{z}$ for $\mathrm{C}_{10} \mathrm{H}_{19} \mathrm{O}_{2}[\mathrm{M}+\mathrm{H}]^{+}=171.1385$; observed $\mathrm{m} / \mathrm{z}=171.1353$.

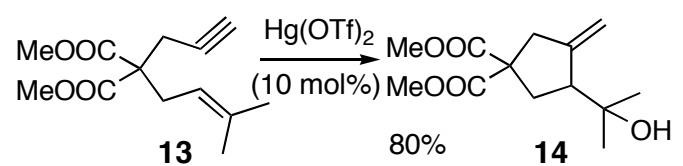

13. ${ }^{1} \mathrm{H}$ NMR (300 MHz, $\left.\mathrm{CDCl}_{3}\right): \delta 4.88-4.82(1 \mathrm{H}, \mathrm{qt}, \mathrm{J}=7.8,1.5 \mathrm{~Hz}), 3.69(6 \mathrm{H}, \mathrm{s}), 2.73(2 \mathrm{H}, \mathrm{d}, \mathrm{J}=7.5 \mathrm{~Hz}), 2.72(2 \mathrm{H}, \mathrm{d}, \mathrm{J}=2.7 \mathrm{~Hz})$, $1.97(1 \mathrm{H}, \mathrm{t}, \mathrm{J}=2.7 \mathrm{~Hz}), 1.66(3 \mathrm{H}, \mathrm{s}), 1.61(3 \mathrm{H}, \mathrm{s}) .{ }^{13} \mathrm{C} \mathrm{NMR}\left(75 \mathrm{MHz}, \mathrm{CDCl}_{3}\right): \delta 170.7,137.2,117.1,79.5,71.4,57.4,52.9,31.0$, 26.3, 22.7, 18.1. Trost, B. M.; Braslau, R. Tetrahedron Lett. 1988, 29, 1231-1234; Mook, R. Jr.; Sher, P. M. Org. Synth. 1988, 66, 7586.

14. ${ }^{1} \mathrm{H}$ NMR (300 MHz, $\left.\mathrm{CDCl}_{3}\right)$ : $\delta 5.06(1 \mathrm{H}, \mathrm{m}), 4.99(1 \mathrm{H}, \mathrm{m}), 3.69(3 \mathrm{H}, \mathrm{s}), 3.68(3 \mathrm{H}, \mathrm{s}), 2.83(2 \mathrm{H}, \mathrm{m}), 2.68-2.55(2 \mathrm{H}, \mathrm{m}), 2.01-1.90$ $(1 \mathrm{H}, \mathrm{m}), 1.80(1 \mathrm{H}, \mathrm{bs}), 1.19(3 \mathrm{H}, \mathrm{s}), 1.16(3 \mathrm{H}, \mathrm{s}) .{ }^{13} \mathrm{C} \mathrm{NMR}\left(75 \mathrm{MHz}, \mathrm{CDCl}_{3}\right): \delta 171.77,171.70,148.3,111.1,72.4,58.6,52.6,52.4$, 43.3, 36.0, 27.8, 26.0. Calcd m/z for $\mathrm{C}_{10} \mathrm{H}_{14} \mathrm{O}_{4}\left[\mathrm{M}-\mathrm{CH}_{3} \mathrm{COCH}_{3}\right]^{+}=198.0892$; observed m/z = 198.0881. Mendez, M.; Munoz, M. P.; Nevado, C.; Cardenas, D. J.; Echavarren, A. M. J. Am. Chem. Soc. 2001, 123, 10511-10520; Mendez, M.; Munoz, M. P.; Echavarren, A. M. J. Am. Chem. Soc. 2000, 122, 11549-11550.

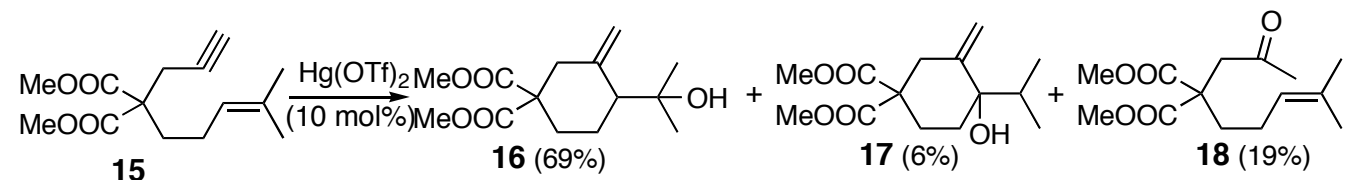

15. ${ }^{1} \mathrm{H}$ NMR (300 MHz, $\left.\mathrm{CDCl}_{3}\right): \delta$ 5.12-5.05 (1H, m), $3.73(6 \mathrm{H}, 2 \mathrm{~s}), 2.84(2 \mathrm{H}, \mathrm{d}, \mathrm{J}=2.7 \mathrm{~Hz}), 2.11-2.03(2 \mathrm{H}, \mathrm{m}), 2.00(1 \mathrm{H}, \mathrm{t}, \mathrm{J}=2.7$ $\mathrm{Hz}), 1.92-1.84(2 \mathrm{H}, \mathrm{m}), 1.67(3 \mathrm{H}, \mathrm{d}, \mathrm{J}=0.9 \mathrm{~Hz}), 1.58(3 \mathrm{H}, \mathrm{bs}) .{ }^{13} \mathrm{C} \mathrm{NMR}\left(50 \mathrm{MHz}, \mathrm{CDCl}_{3}\right): \delta$ 170.6, 132.7, 122.7, 78.8, 71.3, 56.6, 52.7, 32.0, 25.6, 22.8, 22.7, 17.5. Calcd $\mathrm{m} / \mathrm{z}$ for $\mathrm{C}_{14} \mathrm{H}_{23} \mathrm{O}_{4}\left[\mathrm{M}+\mathrm{H}^{+}\right]=253.1440$; observed $\mathrm{m} / \mathrm{z}=253.1449$.

16. ${ }^{1} \mathrm{H}$ NMR (300 MHz, $\left.\mathrm{CDCl}_{3}\right): \delta 4.89(1 \mathrm{H}, \mathrm{bs}), 4.77(1 \mathrm{H}, \mathrm{bs}), 3.60(6 \mathrm{H}, \mathrm{s}), 2.76(1 \mathrm{H}, \mathrm{d}, \mathrm{J}=13.2 \mathrm{~Hz}), 2.62(1 \mathrm{H}, \mathrm{d}, \mathrm{J}=13.2 \mathrm{~Hz})$, 2.24-2.13 (1H, m), 2.06-2.02 (1H, m), 1.93-1.84 (1H, m), 1.76-1.70 (2H, q, J = $6 \mathrm{~Hz}), 1.17(3 \mathrm{H}, \mathrm{s}), 1.14(3 \mathrm{H}, \mathrm{s}) .{ }^{13} \mathrm{C} \mathrm{NMR}(50 \mathrm{MHz}$, $\mathrm{CDCl}_{3}$ ): $\delta 172.0,171.3,143.7,114.6,72.4,56.2,52.4,52.2,51.2,38.6,28.7,28.6,28.2,23.7$. Calcd m/z for $\mathrm{C}_{14} \mathrm{H}_{23} \mathrm{O}_{5}\left[\mathrm{M}^{2} \mathrm{H}^{+}\right]=$ $271.1545 ;$ observed $\mathrm{m} / \mathrm{z}=271.1536$.

17. ${ }^{1} \mathrm{H}$ NMR (300 MHz, $\mathrm{CDCl}_{3}$ ): This material is contaminated with some other product. The following absorptions are characteristic of the title compound. $\delta 5.03(1 \mathrm{H}, \mathrm{t}, \mathrm{J}=0.9 \mathrm{~Hz}), 4.93(1 \mathrm{H}, \mathrm{t}, \mathrm{J}=1.2 \mathrm{~Hz}), 3.73(6 \mathrm{H}, \mathrm{s}), 3.00-2.95(1 \mathrm{H}, \mathrm{dd}, \mathrm{J}=13.8,2.5 \mathrm{~Hz}), 2.63-2.58$ $(1 \mathrm{H}, \mathrm{btd}, \mathrm{J}=13.8 \mathrm{~Hz}), 0.96(3 \mathrm{H}, \mathrm{d}, \mathrm{J}=6.9 \mathrm{~Hz}), 0.80(3 \mathrm{H}, \mathrm{d}, \mathrm{J}=6.9 \mathrm{~Hz})$. 
18. ${ }^{1} \mathrm{H}$ NMR (300 MHz, $\left.\mathrm{CDCl}_{3}\right): \delta 4.99(1 \mathrm{H}, \mathrm{mt}, \mathrm{J}=7.2 \mathrm{~Hz}), 3.68(6 \mathrm{H}, \mathrm{s}), 3.10(2 \mathrm{H}, \mathrm{s}), 2.12(3 \mathrm{H}, \mathrm{s}), 2.02-1.92(2 \mathrm{H}, \mathrm{m}), 1.90-1.78$ $(2 \mathrm{H}, \mathrm{m}), 1.62(3 \mathrm{H}, \mathrm{s}), 1.53(3 \mathrm{H}, \mathrm{s}) .{ }^{13} \mathrm{C}$ NMR $\left(75 \mathrm{MHz}, \mathrm{CDCl}_{3}\right): \delta 205.0,171.1,132.6,122.7,55.0,52.5,46.0,33.4,30.1,25.5,23.3$, 17.4. Calcd $\mathrm{m} / \mathrm{z}$ for $\mathrm{C}_{14} \mathrm{H}_{23} \mathrm{O}_{5}\left[\mathrm{M}+\mathrm{H}^{+}\right]=271.1545$; observed $\mathrm{m} / \mathrm{z}=271.1559$.

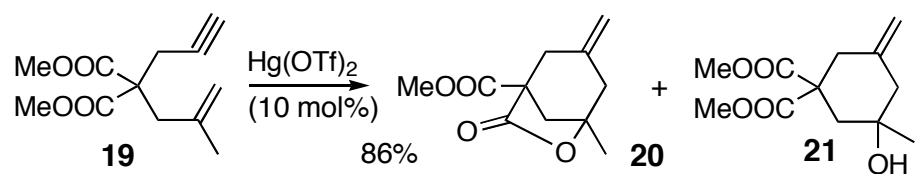

19. ${ }^{1} \mathrm{H}$ NMR (300 MHz, $\left.\mathrm{CDCl}_{3}\right)$ : d $\delta$ 4.92-4.90 (1H, m), 4.85-4.84 (1H, m), $3.74(6 \mathrm{H}, \mathrm{s}), 2.84(2 \mathrm{H}, \mathrm{s}), 2.83(2 \mathrm{H}, \mathrm{d}, \mathrm{J}=2.7 \mathrm{~Hz}), 2.03$ $(1 \mathrm{H}, \mathrm{t}, \mathrm{J}=2.7 \mathrm{~Hz}), 1.65(3 \mathrm{H}, \mathrm{d}, \mathrm{J}=1.2 \mathrm{~Hz}) .{ }^{13} \mathrm{C} \mathrm{NMR}\left(75 \mathrm{MHz}, \mathrm{CDCl}_{3}\right): \delta 170.5,139.7,116.3,79.1,71.7,56.4,52.7,39.5,23.1$, 22.6. Mendez, M.; Munoz, M. P.; Nevado, C.; Cardenas, D. J.; Echavarren, A. M. J. Am. Chem. Soc. 2001, 123, 10511-10520; Gomez, A. M.; Company, M. D.; Valverde, S.; Lopez, J. C. Org. Lett. 2002, 4, 383-386.

20. ${ }^{1} \mathrm{H}$ NMR (300 MHz, $\left.\mathrm{CDCl}_{3}\right): \delta$ 5.06-5.04 $(1 \mathrm{H}, \mathrm{m}), 5.00-4.97(1 \mathrm{H}, \mathrm{m}), 3.79(3 \mathrm{H}, \mathrm{s}), 2.99-2.93(1 \mathrm{H}, \mathrm{md}, \mathrm{J}=15 \mathrm{~Hz}), 2.73-2.67(1 \mathrm{H}$, $\mathrm{td}, \mathrm{J}=11.7,2.4 \mathrm{~Hz}), 2.58-2.51(2 \mathrm{H}, \mathrm{m}), 2,38-2.31(1 \mathrm{H}, \mathrm{qd}, \mathrm{J}=15,2.4 \mathrm{~Hz}), 2.04(1 \mathrm{H}, \mathrm{d}, \mathrm{J}=11.7 \mathrm{~Hz}), 1.53(3 \mathrm{H}, \mathrm{s}) .{ }^{13} \mathrm{C} \mathrm{NMR}(75$ $\mathrm{MHz}, \mathrm{CDCl}_{3}$ ): $\delta$ 173.1, 169.3, 137.7, 117.2, 82.9, 55.6, 52.8, 45.7, 43.3, 38.2, 24.3. Calcd m/z for $\mathrm{C}_{11} \mathrm{H}_{14} \mathrm{O}_{4}=210.0892$; observed $\mathrm{m} / \mathrm{z}=210.0889$.

21. ${ }^{1} \mathrm{H}$ NMR (300 MHz, $\left.\mathrm{CDCl}_{3}\right): \delta 5.03-5.02(1 \mathrm{H}, \mathrm{q}, \mathrm{J}=1.8 \mathrm{~Hz}), 4.93-4.91(1 \mathrm{H}, \mathrm{q}, \mathrm{J}=1.8 \mathrm{~Hz}), 3.73$ (3H, s), $3.72(3 \mathrm{H}, \mathrm{s}), 3.02-2.96$ $(1 \mathrm{H}, \mathrm{td}, \mathrm{J}=13.8,1.8 \mathrm{~Hz}), 2.53-2.46(1 \mathrm{H}, \mathrm{td}, \mathrm{J}=14.4,2.1 \mathrm{~Hz}), 2.32-2.13(4 \mathrm{H}, \mathrm{m}), 2.05-2.00(1 \mathrm{H}, \mathrm{J}=14.4 \mathrm{~Hz}), 1.28(3 \mathrm{H}, \mathrm{s}) .{ }^{13} \mathrm{C}$ NMR (75 MHz, $\left.\mathrm{CDCl}_{3}\right): \delta 172.2,171.7,140.3,114.0,69.9,54.6,52.7,52.5,47.2,42.0,38.5,30.1$. Calcd $\mathrm{m} / \mathrm{z}$ for $\mathrm{C}_{12} \mathrm{H}_{18} \mathrm{O}_{5}$ $=242.1154 ;$ observed $\mathrm{m} / \mathrm{z}=242.1160$.

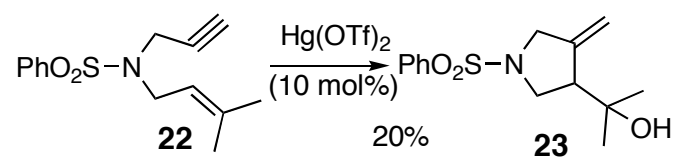

22. ${ }^{1} \mathrm{H}$ NMR (300 MHz, $\left.\mathrm{CDCl}_{3}\right): \delta$ 7.88-7.85 (2H, m), 7.61-7.47 (3H, m), 5.14-5.07 (1H, m), 4.08 (2H, d, J = 2.4 Hz), $3.84(2 \mathrm{H}, \mathrm{d}, \mathrm{J}$ $=7.2 \mathrm{~Hz}), 1.96(1 \mathrm{H}, \mathrm{t}, \mathrm{J}=2.4 \mathrm{~Hz}), 1.73(3 \mathrm{H}, \mathrm{d}, \mathrm{J}=0.9 \mathrm{~Hz}), 1.68(3 \mathrm{H}, \mathrm{d}, \mathrm{J}=0.9 \mathrm{~Hz}) .{ }^{13} \mathrm{C} \mathrm{NMR}\left(75 \mathrm{MHz}, \mathrm{CDCl}_{3}\right): \delta 139.2,139.1$, 132.6, 128.8, 127.7, 117.7, 89.4, 73.3, 44.0, 35.4, 25.9, 17.8. Calcd $\mathrm{m} / \mathrm{z}$ for $\mathrm{C}_{14} \mathrm{H}_{18} \mathrm{NO}_{2} \mathrm{~S}\left[\mathrm{M}+\mathrm{H}^{+}\right]=264.1058$; observed $\mathrm{m} / \mathrm{z}=$ 264.1055. Cao, P.; Wang, B.; Zhang, X. J. Am. Chem. Soc. 2000, 122, 6490-6491.

23. ${ }^{1} \mathrm{H}$ NMR (300 MHz, $\left.\mathrm{CDCl}_{3}\right): \delta$ 7.85-7.81 (2H, m), 7.65-7.52 (3H, m), 5.12-5.08 (2H, m), 3.94-3.87 (1H, qd, J = 13.8, $\left.1.5 \mathrm{~Hz}\right)$, 3.78-3.72 (1H, td, J = 13.8, 1.8 Hz), 3.49-3.44 (1H, dd, J = 10.2, 3.3 Hz), 3.31-3.25 (1H, dd, J = 102, 7.8 Hz), 2.69-2.64 (1H, m), $1.70(1 \mathrm{H}, \mathrm{bs}), 1.17(3 \mathrm{H}, \mathrm{s}), 1.16(3 \mathrm{H}, \mathrm{s}) .{ }^{13} \mathrm{C} \mathrm{NMR}\left(75 \mathrm{MHz}, \mathrm{CDCl}_{3}\right): \delta 144.59,135.5,132.9,129.0,127.7,111.2,71.9,53.8,52.8$, 50.0, 27.0, 26.9. Calcd $\mathrm{m} / \mathrm{z}$ for $\mathrm{C}_{14} \mathrm{H}_{20} \mathrm{NO}_{3} \mathrm{~S}[\mathrm{M}+\mathrm{H}]^{+}=282.1164$; observed $\mathrm{m} / \mathrm{z}=282.1168$. 

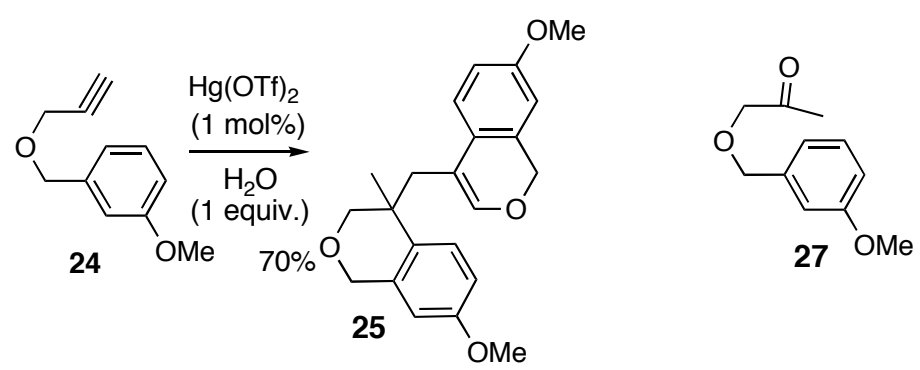

24. ${ }^{1} \mathrm{H}$ NMR $\left(300 \mathrm{MHz}, \mathrm{CDCl}_{3}\right): \delta$ 7.28-7.23 $(1 \mathrm{H}, \mathrm{m}), 6.94-6.91(2 \mathrm{H}, \mathrm{m}), 6.85-6.82(1 \mathrm{H}, \mathrm{m}), 4.58(3 \mathrm{H}, \mathrm{s}), 4.17(2 \mathrm{H}, \mathrm{dd}, \mathrm{J}=2.4,0.9$ $\mathrm{Hz}), 3.80(2 \mathrm{H}, \mathrm{d}, \mathrm{J}=0.6 \mathrm{~Hz}), 2.46(1 \mathrm{H}, \mathrm{dt}, \mathrm{J}=2.4,0.9 \mathrm{~Hz}) .{ }^{13} \mathrm{C} \mathrm{NMR}\left(75 \mathrm{MHz}, \mathrm{CDCl}_{3}\right): \delta 159.7,138.8,129.4,120.2,113.6,113.2$, 79.6, 74.6, 71.3, 57.0, 55.1. Calcd $\mathrm{m} / \mathrm{z}$ for $\mathrm{C}_{11} \mathrm{H}_{12} \mathrm{O}_{2}=176.0837$; observed $\mathrm{m} / \mathrm{z}=176.0834$. Ishikawa, T.; Okano, M.; Aikawa, T.; Saito, S. J. Org. Chem. 2001, 66, 4635-4642; Montevecchi, P. C.; Navacchia, M. L. J. Org. Chem. 1998, 63, 537-542.

25. ${ }^{1} \mathrm{H}$ NMR (300 MHz, $\left.\mathrm{CDCl}_{3}\right): \delta 7.22(1 \mathrm{H}, \mathrm{d}, \mathrm{J}=8.4 \mathrm{~Hz}), 7.13(1 \mathrm{H}, \mathrm{d}, \mathrm{J}=8.4 \mathrm{~Hz}), 6.76-6.73(1 \mathrm{H}, \mathrm{dd}, \mathrm{J}=8.7,2.7 \mathrm{~Hz}), 6.73-6.69$ $(1 \mathrm{H}, \mathrm{dd}, \mathrm{J}=8.7 .2 .4 \mathrm{~Hz}), 6.58(1 \mathrm{H}, \mathrm{d}, \mathrm{J}=2.7 \mathrm{~Hz}), 6.48(1 \mathrm{H}, \mathrm{d}, \mathrm{J}=2.4 \mathrm{~Hz}), 6.33(1 \mathrm{H}, \mathrm{s}), 4.92(2 \mathrm{H}, \mathrm{s}), 4.85-4.73(2 \mathrm{H}, \mathrm{dd}, \mathrm{J}=21.6$, $15.3 \mathrm{~Hz}), 3.82(1 \mathrm{H}, \mathrm{d}, \mathrm{J}=11.4 \mathrm{~Hz}), 3.78(3 \mathrm{H}, \mathrm{s}), 3.75(3 \mathrm{H}, \mathrm{s}), 3.38(1 \mathrm{H}, \mathrm{d}, \mathrm{J}=11.4 \mathrm{~Hz}), 2.73(1 \mathrm{H}, \mathrm{d}, \mathrm{J}=14.4 \mathrm{~Hz}), 2.59(1 \mathrm{H}, \mathrm{d}, \mathrm{J}=$ $14.4 \mathrm{~Hz}), 1.13(3 \mathrm{H}, \mathrm{s}) .{ }^{13} \mathrm{C} \mathrm{NMR}\left(75 \mathrm{~Hz}, \mathrm{CDCl}_{3}\right): \delta 158.3,157.6,143.6,134.8,134.2,129.9,127.4,125.4,122.2,112.7,112.5,110.2$, 108.4, 73.8, 69.0, 68.6, 55.3, 55.2, 37.5, 36.5, 22.9. Calcd m/z for $\mathrm{C}_{22} \mathrm{H}_{24} \mathrm{O}_{4}=352.1675$; observed m/z = 352.1669.

27. ${ }^{1} \mathrm{H}$ NMR (300 MHz, $\left.\mathrm{CDCl}_{3}\right): \delta$ 7.29-7.23 (1H, dt, J = 7.5, $\left.0.9 \mathrm{~Hz}\right), 6.93-6.90(2 \mathrm{H}, \mathrm{m}), 6.86-6.82(1 \mathrm{H}, \mathrm{m}), 4.56(2 \mathrm{H}, \mathrm{s}), 4.05(2 \mathrm{H}$, s), 3,79 (3H, s), $2.14(3 \mathrm{H}, \mathrm{s}) .{ }^{13} \mathrm{C}$ NMR (75 MHz, $\mathrm{CDCl}_{3}$ ): $\delta$ 206.3, 159.6, 138.6, 129.3, 113.3, 112.9, 75.0, 72.9, 55.0, 26.2. Calcd $\mathrm{m} / \mathrm{z}$ for $\mathrm{C}_{11} \mathrm{H}_{14} \mathrm{O}_{3}=194.0943 ;$ Observed $\mathrm{m} / \mathrm{z}=194.0932$.
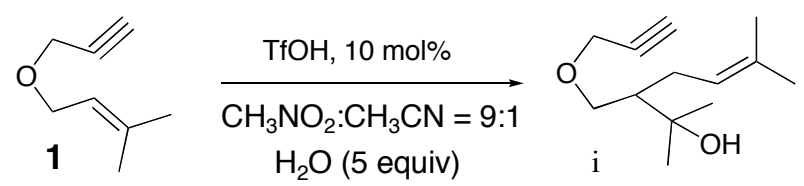

$10 \%$

i: ${ }^{1} \mathrm{H}$ NMR (300 MHz, $\left.\mathrm{CDCl}_{3}\right): \delta$ 5.15-5.08 (1H, mt, J = 7.2, $\left.0.9 \mathrm{~Hz}\right), 4.12(2 \mathrm{H}, \mathrm{d}, \mathrm{J}=2.1 \mathrm{~Hz}), 3.74-3.69(1 \mathrm{H}, \mathrm{dd}, \mathrm{J}=9.6,3.6 \mathrm{~Hz})$, 3,62-3.56 (1H, dd, J = 9.6, $6.3 \mathrm{~Hz}), 3.18(1 \mathrm{H}, \mathrm{bs}), 2.43(1 \mathrm{H}, \mathrm{t}, \mathrm{J}=2.1 \mathrm{~Hz}), 2.17-1.95(1 \mathrm{H}, \mathrm{m}), 1.70(3 \mathrm{H}, \mathrm{bs}), 1.67-1.59(1 \mathrm{H}, \mathrm{m}), 1.61$ (3H, bs), 1.27 (3H, s), $1.20(3 \mathrm{H}, \mathrm{s}) .{ }^{13} \mathrm{C}$ NMR (75 MHz, $\left.\mathrm{CDCl}_{3}\right): \delta 133.2,123.3,79.4,75.0,73.2,70.8,58.7,48.8,29.3,26.5,26.06$, 26.05, 18.0. Calcd m/z for $\mathrm{C}_{13} \mathrm{H}_{21} \mathrm{O}_{2}[\mathrm{M}-\mathrm{H}]^{+}=209.1542$; Observed $\mathrm{m} / \mathrm{z}=209.1534$. 